Pacific Journal of Mathematics

THE NON-MINIMALITY OF INDUCED CENTRAL 


\section{THE NON-MINIMALITY OF INDUCED CENTRAL REPRESENTATIONS}

\section{WRIGHT}

Let $G$ be a finite $p$-group and \&s a minimal faithful permutation representation of $G$ possessing the minimal number of generators of the centre of $G$ transitive constituents. One surmises that the induced representation, 'S', of the centre of $G$, is minimal. The conjecture is validated subject to either of the hypotheses $|G| \leqq p^{5}$ except $G=Q_{8} \times Z_{4}$ or $Z(G) \cong n$ copies of the cyclic group of order $p^{m}$ and is trivial when $G$ is abelian. However, a group of order $p^{6}$ shows the conjecture to be false for $p$ odd, also. The converse problem of extending minimal representations of $Z(G)$ to minimal representations of $G$ is also, in general, not possible.

Notation. $G$ a finite group, $Z(G)$ is the centre of $G, d(Z(G))$ is the minimal number of generators of $Z(G)$. When $G$ is a $p$-group $\Omega_{1}(G)=\left\langle g \in G \mid g^{p}=e\right\rangle . \quad Z p^{m}$ is the cyclic group of order $p^{m} . \quad \mu(G)$ is the least natural number $n$ such that $G$ can be embedded in the symmetric group of degree $n$.

Let $\mathbb{B}=\left\{G_{1}, \cdots, G_{n}\right\}$ be a collection of subgroups of a finite group $G$ and $X_{i}$ be the set of distinct cosets of $G_{i}$ in $G$. The transitive action of $G$ on $X_{i}$ defines a permutation representation of $G$ on the set $X=\bigcup_{i=1}^{n} X_{i}$ with kernel core $\left(\bigcap_{i=1}^{n} G_{i}\right)$. A faithful representation is called minimal in case $|X|=\sum_{i=1}^{n}\left|G: G_{i}\right|$ is minimal over all faithful (S. Suppose now that $G$ is a $p$-group and $d=$ $d(Z(G))$. Then by [1] Theorem $3 n=d$ for $p \neq 2$ whilst when $p=$ $21 / 2 d \leqq n \leqq d$, the upper bound being attained. It is assumed throughout that $n=d$ thereby imposing a restriction on \& only when $p=2$.

The problem is approached by first classifying minimal representations 8 , say, of finite abelian $p$-groups (with a restriction on $\mathbb{B}$ if $p=2$ ) and then observing two elementary properties regarding the structure of $G_{i} \cap Z(G)$.

1. Minimal representations of abelian groups.

THEOREM 1. Let $G$ be a finite abelian p-group with $n \geqq 2$. Suppose $\mathbb{B}=\left\{G_{1}, \cdots, G_{n}\right\}$ is a minimal faithful permutation representation of $G$ and $K_{i}=\bigcap_{\substack{j=1 \\ j \neq i}}^{n} G_{j}$, then

$$
G=\stackrel{x}{x=1}_{i=1}^{n} K_{i} \text { and } \quad G_{i}=\prod_{\substack{j=1 \\ j \neq i}}^{n} K_{j}
$$


Note. Any 85 of this form is a minimal representation of $G$, so this theorem characterizes minimal representations of abelian $p$ groups, $p \neq 2$.

Proof. If $G=Z_{1} \times \cdots \times Z_{n}$ with $Z_{i}$ cyclic then we know that the $G_{i}$ can be reordered so that $G_{i} \cap Z_{i}=E$ (see [2], Lemma 2). Hence $\left|G: G_{i}\right| \geqq\left|Z_{i}\right|$. Suppose for some $k,\left|G: G_{k}\right|>\left|Z_{k}\right|$, then

$$
\mu(G)=\sum_{i=1}^{n}\left|G: G_{i}\right|>\sum_{i=1}^{n}\left|Z_{i}\right|=\mu(G)
$$

so that $\left|G: G_{i}\right|=\left|Z_{i}\right|$, for all $1 \leqq i \leqq n$. Now

$$
\begin{aligned}
\left|G: K_{i}\right| & =\left|G: \bigcap_{\substack{j=1 \\
j \neq i}}^{n} G_{j}\right| \leqq \prod_{\substack{j=1 \\
j \neq i}}^{n}\left|G: G_{j}\right|, \text { Pointcaré's theorem } \\
& =\prod_{\substack{j=1 \\
j \neq i}}^{n}\left|Z_{j}\right|=\left|G: Z_{i}\right| .
\end{aligned}
$$

It follows that $\left|K_{i}\right| \geqq\left|Z_{i}\right|$ and $\left|X_{i=1}^{n} K_{i}\right| \geqq \prod_{i=1}^{n}\left|Z_{i}\right|=|G|$ so that $G=\times_{i=1}^{n} K_{i}$ and $\left|K_{i}\right|=\left|Z_{i}\right|$ (see [3], Lemma 0). Also, $G_{i} \supseteqq \prod_{\substack{j=1 \\ i \neq i}}^{n} K_{j}$ but $\left|G: \prod_{\substack{j=1 \\ j \neq i}}^{n} K_{j}\right|=\left|K_{i}\right|=\left|Z_{i}\right|=\left|G: G_{i}\right|$ and the lemma is now clear.

From the proof of [1], Proposition 2 we conclude that whenever $G$ and $H$ have coprime orders any stabilizer in a minimal representation of $G \times H$ has the form $G_{1} \times H$ or $G \times H_{1}, G_{1} \leqq G, H_{1} \leqq H$. By decomposing an abelian group $A$ into the direct product of its Sylow $p$-subgroups we easily generalize Theorem 1 to classify minimal representations of abelian groups (of odd order).

2. Induced central representation. Throughout this section whenever \& $=\left\{G_{1}, \cdots, G_{n}\right\}, n=d(Z(G))$.

Lemma 2. No generator of $G_{i} \cap Z(G)$ is a p-power of any element in $Z(G)$ provided \&s is minimal.

Proof. Let $H_{i}=\left(\bigcap_{\substack{j=1 \\ j \neq i}}^{n} G_{j}\right) \cap Z(G)$. Since $G_{i} \supseteqq H_{1} \times \cdots \times H_{i-1} \times$ $H_{i+1} \times \cdots \times H_{n}$, see [3] lemma, it follows that $d\left(G_{i} \cap Z(G)\right)=n-1$. Suppose $G_{i} \cap Z(G)=\left\langle x_{k} \mid k \in I\right\rangle$ and $x_{j}=y^{p}$, for some $j$. Then $|I| \geqq$ $n-1$. Define $Y=\left\langle x_{k}, y \mid k \in I \backslash\{j\}\right\rangle \supseteqq G_{i} \cap Z(G)$. Clearly, $\Omega_{1}(Y)=$ $\Omega_{1}\left(G_{i} \cap Z(G)\right)$ and $Y G_{i} \cap Z(G)=Y$. Thus, the representation $\left\{G_{1}, \cdots\right.$, $\left.G_{i-1}, Y G_{i}, G_{i+1}, \cdots, G_{n}\right\}$ is faithful. The minimality of \&S yields $Y G_{i}=$ $G_{i}$ so that $Y=G_{i} \cap Z(G)$. It follows that $x_{j} \in\left\langle x_{k} \mid k \in I \backslash\{j\}\right\rangle$, contradicting that $x_{i}$ is a generator of $G$.

The next lemma is easy to verify. 
LEMMA 3. Let $A=\mathrm{X}_{i=1}^{n}\left\langle a_{i}\right\rangle$ be an abelian p-group with $d(A)=$ n. If $B \leqq A$ with $d(B)=n-1$ such that no generator of $B$ is a p-power of any element of $A$ then

or

(i) $B=\left\langle a_{j}\right| j \in N \backslash\{s\}$, some $\left.s\right\rangle$, where $N=\{k \mid 1 \leqq k \leqq n\}$

(ii) $B=\left\langle a_{r} a_{s}^{\gamma r}, a_{k}\right| r \in J, k \in K, J \cup K=N \backslash\{s\}$, some $\left.s, J \cap K=\Phi\right\rangle$.

Corollary. If $Z(G)=Z_{1} \times \cdots \times Z_{n}$ with $Z_{i}=\left\langle z_{i}\right\rangle$ cyclic then $G_{i} \cap Z(G)=\left\langle z_{j} \mid j \in N \backslash\{s\}\right\rangle$

or

$G_{i} \cap Z(G)=\left\langle z_{r} z_{s}^{\gamma}, z_{k} \mid r \in J, k \in K, J \cup K=N \backslash\{s\}, J \cap K=\Phi\right\rangle$.

Proof. By Lemma $2 G_{i} \cap Z(G)$ and $Z(G)$ satisfy the conditions of Lemma 3.

Write (S) $^{\prime}=\left\{G_{1} \cap Z(G), \cdots, G_{n} \cap Z(G)\right\}$ then:

LEMma 4. 'S' is minimal whenever $Z(G) \cong n$ copies of $Z_{p}^{m}$.

Proof. $n=1$ is trivial. For $n \neq 1$, by the corollary to Lemma 3 we deduce $\left|Z: G_{i} \cap Z(G)\right|=p^{m}, 1 \leqq i \leqq n$, yielding deg $\mathbb{S}^{\prime}=n p^{m}$ and $\left.{ }^{\prime \prime}\right)^{\prime}$ is minimal.

THEOREM 5. If $|G| \leqq p^{5}$ then (\$3' is minimal, except for the case $p=2, G=Q_{8} \times Z_{4}$, the direct product of the quaternionic group of order 8 and the cyclic group of order 4.

Proof. We already have the result if $G$ is abelian or $Z(G)$ is isomorphic to $n$ copies of $Z_{p}^{m}$. This leaves the case: $|G|=p^{5}$, $Z(G)=\left\langle z_{1}\right\rangle \times\left\langle z_{2}\right\rangle \cong Z_{p 2} \times Z_{p}$. If $G=H \times K$ and is non-abelian then $K \cong Z_{p}$ or $K \cong Z_{p 2}$. Let $\mathbb{S}=\left\{G_{1}, G_{2}\right\}$ be a minimal faithful representation of $G$. By [3], $\mu(G)=\mu(H)+\mu(K)$. When $K \cong Z_{p}$, $\left|G: G_{1}\right|=p$, say, and $G_{1} \cap Z(H) \neq E$. By the corollary to Lemma 3, $G_{1} \supseteq Z(H)$, so that (S) $^{\prime}$ is minimal. If $K \cong Z_{p^{2}}$, then except for the case $p=2$ and $H \cong Q_{8}, \mu(H)=p^{2}$. Therefore, $\mu(G)=p^{2}+p^{2}$ and $\left|G_{1}\right|=\left|G_{2}\right|=p^{3}$. As above, (S)' not minimal implies $G_{1} \cap Z(H)=E=$ $G_{2} \cap Z(H)$. It follows that $G=G_{1} Z(H)=G_{2} Z(H)$ and $G_{1}, G_{2}$ are normal subgroups of $G$. Hence, $G_{1} \cap G_{2}$ is a nontrivial normal subgroup of $G$, contradicting the faithfulness of $\mathbb{S}$. When $G=Q_{8} \times Z_{4}$, suppose $Q_{8}=\left\langle x, y \mid x^{2}=y^{2}, x^{y}=x^{-1}\right\rangle, \quad Z_{4}=\left\langle z \mid z^{4}=e\right\rangle$. Then (S) $=$ $\left\{Q_{8},\langle x z\rangle\right\}$ is minimal but $\mathcal{B S}^{\prime}=\left\{\left\langle x^{2}\right\rangle,\left\langle x^{2} z^{2}\right\rangle\right\}$ is not. Under the hypothesis $G \not \equiv Q_{8} \times Z_{4}$, (a) any counterexample is not a nontrivial direct 
product. We also have, (b) $g^{p}$ is central for all $g \in G$, since $G / Z \cong$ $Z_{p} \times Z_{p}$. By Lemma 2, since $\left|G_{1} \cap Z(G)\right|=p=\left|G_{2} \cap Z(G)\right|$, we may assume without loss of generality that $G_{1} \cap Z(G)=\left\langle z_{2}\right\rangle, G_{2} \cap Z(G)=$ $\left\langle z_{1}^{p r} z_{2}\right\rangle$ where $(r, p)=1$ because $G_{i} \supseteqq\left\langle z_{1}^{p}\right\rangle$ implies $G_{i} \supseteqq\left\langle z_{1}\right\rangle$. Also, if $\left|G_{i}\right|=p^{3}$ then $G_{i} \cap Z_{p^{2}}=E$ yields $G=G_{i} Z_{p 2}$ : Let $g \in G_{i}, h \in G$ then $h=g_{1} z, g_{1} \in G_{i}, z \in Z_{p^{2}}$ hence $g^{h}=g^{g_{1} z}=g^{g_{2}} \in G_{i}$ so $G_{i}$ is normal in $G$ and $G=G_{i} \times Z_{p 2}$, contradicting (a). We deduce, (c) $\left|G_{i}\right| \leqq p^{2}, i=$ 1,2 and $\mu(G) \geqq 2 p^{3}$.

Let $M$ be a maximal subgroup of $G$ containing $Z(G)$, then $M$ is abelian and has one of the forms:
(i) $M=\langle a\rangle \times\langle b\rangle \times\langle c\rangle \cong Z_{p^{2}} \times Z_{p} \times Z_{p}$,
(ii) $M=\langle a\rangle \times\langle b\rangle \cong Z_{p 3} \times Z_{p}$,
(iii) $M=\langle a\rangle \times\langle b\rangle \cong Z_{p^{2}} \times Z_{p^{2}}$.

Case (i). We can choose $a, b, c$ so that $Z(G)=\langle a\rangle \times\langle b\rangle$ and then $[\langle a, c\rangle \cap\langle b, c\rangle] \cap Z(G)=\langle c\rangle \cap Z(G)=E$ giving $\mu(G) \leqq p^{2}+p^{3}\left\langle 2 p^{3}\right.$, contradicting (c). Case (ii). $Z(G)=\left\langle a^{p}\right\rangle \times\langle b\rangle$. Suppose $G / M=\langle c M\rangle$. $c^{p}=e$ implies case (i) holds. $c^{p} \neq e$ then $c^{p}=a^{p r} b^{s}$ by (b). If $p \mid r$, let $c_{1}=c a^{-r} \notin M$ then $c_{1}^{p}=b^{s}$ and $\left\{\langle a\rangle,\left\langle c_{1}, b\right\rangle\right\}$ is faithful of degree less than $2 p^{3}$. Hence for all $c \in G \backslash M\langle c\rangle \cap\langle a\rangle \neq E$. Let $\$ S=\left\{G_{1}, G_{2}\right\}$ be minimal then by Lemma $2, G_{2} \cap\langle a\rangle=E$ and it follows that $\left|G_{i}\right|=$ $p$, contradicting the minimality of \&s. Case (iii). Without loss of generality we may assume $Z(G)=\langle a\rangle \times\left\langle b^{p}\right\rangle$. Suppose $G / M=\langle c M\rangle$. $c^{p}=e$ implies case (i) holds. If $c^{p 2} \neq e$ then $\langle c\rangle \cap\langle a\rangle=E$ or $\langle c\rangle \cap$ $\langle b\rangle=E$ so that $|c|=p^{3}$ and $\{\langle c\rangle,\langle a\rangle\}$ or $\{\langle c\rangle,\langle b\rangle\}$ is faithful of degree less than $2 p^{3}$. This leaves the case $c^{p 2}=e . c^{p}$ is central, $c^{p}=a^{p r} b^{p s}$, say, but $\left(c a^{-r}\right)^{p}=b^{p s}$ and $c a^{-r} \notin M$. As above, $b^{p s}=e$ reduces to case (i). We may now assume that

$G=\left\langle a, b, c \mid a^{p 2}=b^{p 2}=c^{p 2}=e=[a, b]=[a, c], b^{p}=c^{p},[b, c]=a^{p u} b^{p v}\right\rangle$.

If $a^{p u}=e$ then $G$ is a nontrivial direct product. If $b^{p v} \neq e$ we can choose $a$ so that $[b, c]=\left(a^{p} b^{p}\right)^{v}$ then $[a b, a c]=[b, c]=(a b)^{p v}$ but $G=$ $\langle a, a b, a c\rangle$ and we proceed as above. By suitable choice of $a$ it remains to eliminate the case $[b, c]=a^{p}$. Since $\left(b^{-1} c\right)^{p}=[b, c]^{-1 / 2 p(p+1)}$, when $p \neq 2\left(b^{-1} c\right)^{p}=e$ and when $p=2\left(a b^{-1} c\right)^{2}=e$. In either case $G / M$ can be generated by an element of order $p$. This completes the argument.

While attacking groups of order $p^{6}$ by identical methods to Theorem 5, one obtains the following counterexample.

Theorem 6. Let $G=\langle a, b, c| a^{p^{3}}=b^{p^{2}}=c^{p}=1=[a, b]=[a, c]$, $[c, b]=a^{p^{2}}>$ then

(i) $|G|=p^{8}$ and $Z(G)=\langle a\rangle \times\left\langle b^{p}\right\rangle \cong Z_{p^{3}} \times Z_{p}$,

(ii) $G$ is not a nontrivial direct product,

(iii) $\mu(G)=p^{2}+p^{4}$, 
(iv) $\mathbb{S}=\{\langle a b, c\rangle,\langle b\rangle\}$ is a minimal representation of $G$, but BS' $^{\prime}=\{\langle a b, c\rangle \cap Z(G),\langle b\rangle \cap Z(G)\}$ is not minimal.

Proof. (i) For $1 \leqq i \leqq p^{2}$ define $\alpha_{\imath}, \beta_{i}, \gamma_{i}$ by

$$
\begin{aligned}
& \alpha_{i}:(r, i, s) \mapsto(r, i, s+1) \\
& \beta_{i}:(r, i, s) \mapsto(r+s, i, s+2) \\
& \gamma_{i}:(r, i, s) \mapsto(r+1, i, s)
\end{aligned}
$$

$1 \leqq r, s \leqq p, \bmod p$ in the first and third components [i.e., $\alpha_{1}=$ $((1,1,1)(1,1,2) \cdots(1,1, p))((2,1,1)(2,1,2) \cdots(2,1, p)) \cdots((p, 1,1) \cdots$ $(p, 1, p))] . \quad \alpha_{i}, \beta_{i}, \gamma_{i}$ each have order $p$ and $\left[\alpha_{i}, \beta_{i}\right]=\gamma_{i}$. Define $\lambda$, $\mu, \nu$ as follows

$$
\begin{aligned}
\lambda:(r, i, s) & \mapsto\left\{\begin{array}{l}
(r, i+1, s), 1 \leqq i \leqq p^{2} \\
(r+1,1, s), i=p^{2}
\end{array}\right. \\
\mu & =\left(12 \cdots p^{2}\right) \prod_{i=1}^{p^{2}} \beta_{i} \\
\nu & =\prod_{i=1}^{p^{2}} \alpha_{\imath} .
\end{aligned}
$$

$\lambda, \mu, \nu$ satisfy $\lambda^{p^{3}}=\mu^{p^{2}}=\nu^{p}=1=[\lambda, \mu]=[\lambda, \nu],[\nu, \mu]=\lambda^{p^{2}}$. Clearly any element of $G$ has the form $a^{i} b^{j} c^{k}, 0 \leqq i<p^{3}, 0 \leqq j<p^{2}, 0 \leqq k<$ $p$ and the representation shows that these are distinct and (i) follows.

(ii) Suppose $G=H \times K$, then $Z(G)=Z(H) \times Z(K)$. We may assume $Z(H) \cong Z_{p}$ and $Z(K)=\left\langle a b^{p s}\right\rangle \cong Z_{p^{3}} . \quad K \cap\langle b\rangle=E$ implies $|K| \leqq p^{4}$. If $|K|=p^{4} K$ and $H$ are abelian and consequently $G$ is abelian. It follows that $|K|=|H|=p^{3}$. Therefore, there exist $h \in H$ and $r, 0 \leqq r<p^{3}$ such that $c=\left(a b^{p s}\right)^{r} h$ then $[h, b]=\left[\left(a b^{p s}\right)^{r} h, b\right]$ (since $\left(a b^{p s}\right)^{r}$ is central) $=[c, b]=a^{p^{2}}$. But $H$ is normal in $G$ and so $a^{p^{2}}=[h, b] \in H \cap K$, a contradiction.

(iii) Let $\mathbb{S}=\left\{G_{1}, G_{2}\right\}$ be a minimal faithful representation of $G$. This always exists by [1], Theorem 3. If $\left|G: G_{i}\right|=p$ then $G_{2}$ is normal in $G$ and $G$ is a nontrivial direct product. Therefore, $\left|G: G_{i}\right| \geqq p^{2}, i=1,2$. For some $i, G_{i} \cap\langle a\rangle=E$, since (5) is faithful suppose, say, $G_{1} \cap\langle a\rangle=E$. If $\left|G_{1}\right|=p^{3}, G=G_{1} \times\langle a\rangle$ since $a$ is central. Hence $\mu(G) \geqq p^{2}+p^{4}$ but (i) exhibits a faithful representation of degree $p^{2}+p^{4}$. The final part of the theorem is now easy.

The converse problem: Given $\mathbb{B S}^{\prime}=\left\{Z_{1}, \cdots, Z_{n}\right\}, \quad n=d(Z(G))$ a minimal representation of $Z(G)$, does there exist a minimal representation $\mathbb{S}=\left\{G_{1}, \cdots, G_{n}\right\}$ of $G$ such that $G_{i} \cap Z(G)=Z_{i}$ ? The answer to this question is quickly found to be negative. 
Lemma 7. Let $G=H \times K$ where $H=\left\langle a, b \mid a^{p}=b^{p}=[a, b]\right\rangle$ and $K=\left\langle c \mid c^{p}=e\right\rangle$ then BS' $^{\prime}=\left\{\left\langle a^{p} c\right\rangle,\langle c\rangle\right\}$ is a minimal representation of $Z(G)$ which cannot be extended to a minimal representation of $G$.

Proof. When $p \neq 2 H$ is the non-abelian group of order $p^{3}$ containing an element of order $p^{2}$ and when $p=2 H$ is the quaternionic group of order 8. $Z(H)=\left\langle a^{p}\right\rangle$ and (S' $^{\prime}$ is obviously minimal. Now

$$
\begin{aligned}
\left(a^{i} b^{j}\right)^{p} & =b^{j p}\left(b^{-j p} a^{i} b^{j p}\right)\left(b^{-j(p-1)} a^{i} b^{j(p-1)}\right) \cdots\left(b^{-j} a^{i} b^{j}\right), \quad j \neq 0 \\
& =a^{(i+j) p+i j^{p}(1+\cdots+p)}, \text { since } a^{b}=a^{p+1},\left(a^{i}\right)^{b^{m}}=a^{i(m p+1)} .
\end{aligned}
$$

Case I. $p \neq 2$ then $p \mid(1+\cdots+p)=1 / 2 p(p+1)$ and

$$
\left(a^{i} b^{j} c^{k}\right)^{p}=a^{(i+j) p} \text { for all } i, j, k \text {. }
$$

Every element of $G$ has the form $a^{i} b^{j} c^{k}, 0 \leqq i<p^{2}, 0 \leqq j, k<p$. If $G_{1} \supseteqq\left\langle a^{p} c\right\rangle$ then $a^{i} b^{j} c^{k} \in G_{1}$ implies that $i+j=0(\bmod p)$ i.e., $j=$ $r p-i$ consequently for each choice of $i$ there is only one choice for $j$. It follows that $\left|G_{1}\right| \leqq p^{2}$ and $\left|G: G_{1}\right| \geqq p^{2}$ since $G_{1} \cap\langle c\rangle=E$. By $(*), \quad\left(a b^{p-1}\right)^{p}=a^{p 2}=e,\left\langle a b^{p-1}\right\rangle \cap Z(H)=E$ and trivially $\mu(H)=p^{2}$. By [3], $\mu(G)=\mu(H)+\mu(K)=p^{2}+p$. $G_{2} \supseteqq\langle c\rangle$ so $Z(H) \cap G_{2}=E$ and $\left\{H, G_{2}\right\}$ is faithful. Therefore, $|G: H|+\left|G: G_{2}\right| \geqq \mu(G)=p^{2}+p$ and $\left|G: G_{2}\right| \geqq p^{2}$. Hence deg $\left\{G_{1}, G_{2}\right\}=\left|G: G_{1}\right|+\left|G: G_{2}\right| \geqq 2 p^{2}>\mu(G)$ proving $\left\{G_{1}, G_{2}\right\}$ is not minimal.

Case II. $p=2, \mu(H)=8$ and $\mu(G)=\mu(H)+\mu(K)=10$, by [3]. (*) becomes

$$
\left(a^{i} b^{j} c^{k}\right)^{2}=a^{(i+j) 2+i j 2}=\left\{\begin{array}{l}
e, i, j \text { both even } \\
a^{2}, \text { otherwise }
\end{array}\right.
$$

One easily checks that $G_{1}=\left\langle a^{2} c\right\rangle, G_{2}=\langle c\rangle$ and $\left.\operatorname{deg}\left\{G_{1}, G_{2}\right\}=16\right\rangle$ $\mu(G)$ which proves the lemma.

\section{REFERENCES}

1. D. L. Johnson, Minimal permutation representations of finite groups, Amer. J. Math., 93 (1971), 857-866.

2. D. Wright, Degrees of minimal permutation representations of covering groups of abelian groups, Amer. J. Math., (to appear).

3. - Degrees of minimal embeddings for some direct products, Amer. J. Math., (to appear).

Received June 12, 1973. May I acknowledge the generous financial assistance of the Science Research Council and the first class advice of Dr. D. L. Johnson.

UNIVERSITY OF NOTTINGHAM

Present address: Australian National University 


\section{PACIFIC JOURNAL OF MATHEMATICS}

\section{EDITORS}

RICHARD ARENS (Managing Editor)

University of California

Los Angeles, California 90024

R. A. Beaumont

University of Washington

Seattle, Washington 98105
J. DugundJI

Department of Mathematics

University of Southern California

Los Angeles, California 90007

D. Gilbarg and J. Milgram

Stanford University

Stanford, California 94305

\section{ASSOCIATE EDITORS}

E. F. BECKENBACH

B. H. NeUMANN

F. WOLF

K. YosHIDA

\section{SUPPORTING INSTITUTIONS}

UNIVERSITY OF BRITISH COLUMBIA

CALIFORNIA INSTITUTE OF TECHNOLOGY

UNIVERSITY OF CALIFORNIA

MONTANA STATE UNIVERSITY

UNIVERSITY OF NEVADA

NEW MEXICO STATE UNIVERSITY

OREGON STATE UNIVERSITY

UNIVERSITY OF OREGON

OSAKA UNIVERSITY
UNIVERSITY OF SOUTHERN CALIFORNIA

STANFORD UNIVERSITY

UNIVERSITY OF TOKYO

UNIVERSITY OF UTAH

WASHINGTON STATE UNIVERSITY

UNIVERSITY OF WASHINGTON

$\stackrel{*}{*} \stackrel{*}{*} \stackrel{*}{*}$ AMERICAN MATHEMATICAL SOCIETY




\section{Pacific Journal of Mathematics}

\section{Vol. 53, No. $1 \quad$ March, 1974}

Martin Bartelt, Strongly unique best approximates to a function on a set, and a finite

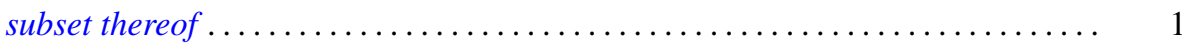

S. J. Bernau, Theorems of Korovkin type for $L_{p}$-spaces $\ldots \ldots \ldots \ldots \ldots \ldots \ldots \ldots \ldots$

S. J. Bernau and Howard E. Lacey, The range of a contractive projection on an

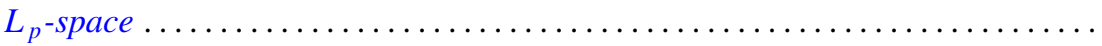

Marilyn Breen, Decomposition theorems for 3-convex subsets of the plane ......... Ronald Elroy Bruck, Jr., A common fixed point theorem for a commuting family of

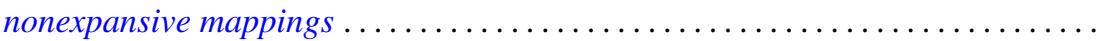

Aiden A. Bruen and J. C. Fisher, Blocking sets and complete $k$-arcs . . . . . . . 73

R. Creighton Buck, Approximation properties of vector valued functions . ......... 85

Mary Rodriguez Embry and Marvin Rosenblum, Spectra, tensor products, and

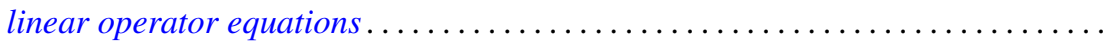

Edward William Formanek, Maximal quotient rings of group rings . . . . . . . . . 109

Barry J. Gardner, Some aspects of T-nilpotence . . . . . . . . . . . . . . . 117

Juan A. Gatica and William A. Kirk, A fixed point theorem for $k$-set-contractions

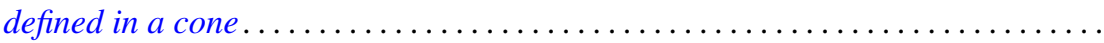

Kenneth R. Goodearl, Localization and splitting in hereditary noetherian prime

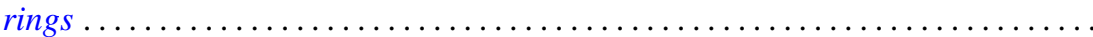

James Victor Herod, Generators for evolution systems with quasi continuous

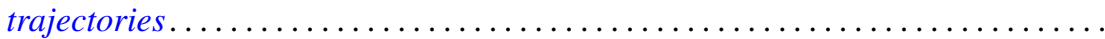

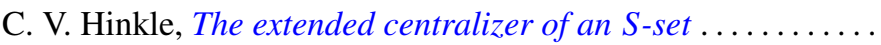

I. Martin (Irving) Isaacs, Lifting Brauer characters of p-solvable groups . . .

Bruce R. Johnson, Generalized Lerch zeta function ...........

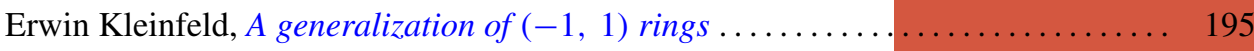

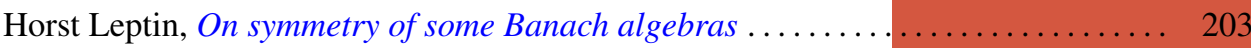

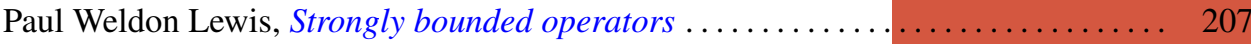

Arthur Larry Lieberman, Spectral distribution of the sum of self-adjoint

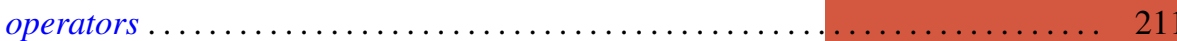

I. J. Maddox and Michael A. L. Willey, Continuous operators on paranormed spaces and matrix transformations

James Dolan Reid, On rings on groups ........................... 229

Richard Miles Schori and James Edward West, Hyperspaces of graphs are Hilbert cubes.

William H. Specht, A factorization theorem for p-constrained groups ...

Robert L Thele, Iterative techniques for approximation of fixed points of certain nonlinear mappings in Banach spaces ...............

Tim Eden Traynor, An elementary proof of the lifting theorem

Charles Irvin Vinsonhaler and William Jennings Wickless, Completely decomposable groups which admit only nilpotent multiplications .

Raymond O’Neil Wells, Jr, Comparison of de Rham and Dolbeault cohomology for

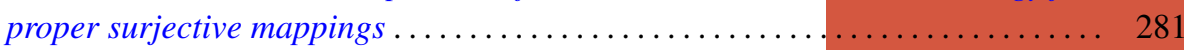

David Lee Wright, The non-minimality of induced central representations . . . . . 301 